\title{
AVALIAÇÃO DA LINGUAGEM DOCUMENTÁRIA DeCS NA ÁREA DE FONOAUDIOLOGIA NA PERSPECTIVA DO USUÁRIO: estudo de observação da recuperação da informação com protocolo verbal EVALUATION OF DeCS INDEXING LANGUAGE IN SPEECH-LANGUAGE PATHOLOGY AND AUDIOLOGY AREA, IN THE USER'S VIEW: an observation study of the information retrieval with a verbal protocol
}

\author{
Vera Regina Casari Boccato \\ Bibliotecária da Faculdade de Odontologia de Bauru Universidade de São Paulo \\ Mestre em Ciência da Informação \\ Universidade Estadual Paulista -UNESP-Marília \\ vboccato@yahoo.com.br \\ Mariângela Spotti Lopes Fujita \\ Professora Doutora do Departamento de Ciência da Informação \\ Universidade Estadual Paulista - UNESP-Marília \\ goldstar@flash.tv.br
}

\section{Resumo}

A proposta deste estudo é avaliar, pela observação do usuário, a linguagem documentária Descritores em Ciências da Saúde (DeCS), na área de Fonoaudiologia, utilizada para a recuperação da informação no sistema de informação da Literatura Latino-Americana e do Caribe em Ciências da Saúde (LILACS), produzido pelo Centro Latino-Americano e do Caribe de Informação em Ciências da Saúde (BIREME), por meio da técnica do protocolo verbal, pelo fato de os pesquisadores da área de Fonoaudiologia necessitarem de um sistema de informação que utilize uma linguagem documentária que represente os valores e a cultura desse sistema em que ela está inserida. Com essa proposição, tem-se por objetivo contribuir para o aperfeiçoamento da linguagem documentária DeCS, visando melhor representatividade terminológica na área da Fonoaudiologia brasileira como fator determinante para o desenvolvimento de pesquisas científicas de qualidade. A abordagem metodológica empregada foi à qualitativa-cognitiva, tendo sido a técnica do protocolo verbal aplicada em pesquisadores (sujeitos) do Departamento de Fonoaudiologia da Faculdade de Odontologia de Bauru da Universidade de São Paulo (FOB-USP), representantes das quatro especialidades formadoras da área: Linguagem, Audiologia, Voz e Motricidade Oral para a realização da coleta de dados. A análise das transcrições desses protocolos demonstrou o bom desempenho da metodologia empregada, bem como possibilitou uma reflexão sobre essas declarações revelando que a linguagem DeCS, em Fonoaudiologia, conduziu as buscas a resultados insatisfatórios quanto à recuperação da informação apontando ocorrências relevantes como a insuficiência da quantidade de termos genéricos e/ou específicos representativos da área de fonoaudiologia; a necessidade da atualização de termos disponíveis na linguagem com relação à terminologia encontrada na literatura científica da área e adotada pelos especialistas; a hierarquização de termos em categorias de assuntos não equivalentes aos seus conceitos, a tradução de diversos termos da língua inglesa para a portuguesa, e desta para a inglesa, não correspondendo ao equivalente utilizado na área de fonoaudiologia, entre outras. Conclui-se que se faz necessário o aprimoramento do DeCS na área de Fonoaudiologia e, para tanto, recomenda-se a BIREME a construção de uma categoria específica nessa área, para a efetiva representação terminológica em consonância com a empregada na literatura e utilizada pela comunidade científica brasileira. 
Palavras-chave: Linguagem documentária. Avaliação - linguagem documentária. Sistema de Informação. Recuperação da informação. Protocolo verbal. Fonoaudiologia.

\section{INTRODUÇÃO}

Nos últimos vinte anos, a comunidade científica vem atribuindo à pesquisa a responsabilidade de ser uma importante ferramenta para a geração do conhecimento que contribui para a melhoria da situação da saúde da população brasileira, bem como nas tomadas de decisão com respeito ao planejamento e programas de saúde e na geração do conhecimento (CONFERÊNCIA NACIONAL DE CIÊNCIA, TECNOLOGIA E INOVAÇÃO EM SAÚDE, 2004).

Contextualizando a área de Fonoaudiologia ${ }^{1}$ dentro do cenário das Ciências da Saúde, esta abrange os principais níveis de atenção primária à saúde: preventivo, curativo e reabilitacional. Considerada uma área multidisciplinar pelas interfaces que possui, principalmente com a Psicologia, Lingüística, Educação, Medicina e Odontologia, o trabalho do profissional fonoaudiólogo é desenvolvido em vários serviços à comunidade. Além disso, a área, também, contribui efetivamente para o desenvolvimento de pesquisas de campo e aplicadas, beneficiando diretamente a comunidade civil.

A Fonoaudiologia, ciência que se destina a cuidar do indivíduo ou de populações no que se refere à comunicação humana em seus aspectos da fala, linguagem oral e escrita, audição e sistema sensório motor oral, promovendo, habilitando, aperfeiçoando e recuperando os padrões comunicativos (ANDRADE, 1996), teve os seus primórdios marcados no final do século XIX, quando a profissão de fonoaudiólogo foi reconhecida na Hungria, mas o seu crescimento técnico e acadêmico-científico ocorreu a partir da década de 1960, quando no Brasil surgiram as duas primeiras faculdades pertencentes, respectivamente, à Universidade de São Paulo (USP) e à Pontifícia Universidade Católica de São Paulo (PUC). O período de 1960 até o início de 1980 foi relevante para o fortalecimento da área, o que possibilitou o reconhecimento da profissão pela Lei n ${ }^{0} 6965$ de 09 de dezembro de 1981. (MARCHESAN; ZORZI, GOMES, 1998).

A partir da década de 1980, a pesquisa científica na área de Fonoaudiologia tomou impulsos notáveis na descoberta de novos conhecimentos, na assistência às tomadas de

\footnotetext{
${ }^{1}$ A palavra Fonoaudiologia teve sua origem terminológica nos vocabulários grego e latim. FONO (do grego) significando som claro e forte, som articulado, linguagem, palavra, expressão; AUDIO (do latim), que significa ouvir, escutar, entender, compreender e LOGIA, (do grego), com o significado de palavra, expressão pensamento, discurso lógico. (CURIOSIDADE: história da fonoaudiologia. Disponível em: $<$ http://www.unig.br/historia_fono.htm>. Acesso em: 21 mar. 2005).
} 
decisões e nas resoluções de problemas, levando o usuário/pesquisador ao encontro de informações úteis e pertinentes à sua realidade investigativa.

Assim, a área de Fonoaudiologia, realiza pesquisas importantes que viabilizam o desenvolvimento científico, com estudos sobre os problemas da comunicação humana, com o intuito de novas descobertas e diretrizes na adoção de técnicas de avaliação, de terapias, de atitudes preventivas, voltadas ao tratamento e/ou reabilitação de pacientes com distúrbios auditivos, de fala e de linguagem, entre outras patologias.

Nesse sentido, as universidades e suas respectivas unidades de informação têm um papel fundamental na geração, disseminação e difusão desse conhecimento.

Um sistema de informação deve refletir as necessidades do usuário/pesquisador na busca de informações que vão ao encontro de suas investigações.

A linguagem documentária, sendo o instrumento de comunicação entre a informação, o sistema de informação e o usuário, deve assegurar o acesso a essa informação, possibilitando sua adequada recuperação e, conseqüentemente, a criação desse conhecimento científico, para promover o bem-estar da sociedade.

Entretanto, se essa linguagem documentária não se apresenta em concordância com as solicitações de buscas realizadas pelo usuário, torna-se, muitas vezes, questionável quanto à qualidade da pesquisa realizada e à credibilidade do Sistema de Informação.

A proposição deste estudo é, portanto, avaliar, pela observação do usuário, a linguagem documentária Descritores em Ciências da Saúde (DeCS), na área de Fonoaudiologia, do sistema de informação Literatura Latino-Americana e do Caribe em Ciências da Saúde (LILACS), produzido pelo Centro Latino-Americano e do Caribe de Informação em Ciências da Saúde (BIREME), com a aplicação da técnica do protocolo verbal ou "pensar alto" (thinking aloud) com o objetivo de contribuir para o levantamento de indicadores que nortearão as estratégias de aprimoramento da linguagem em Fonoaudiologia.

\section{O SISTEMA DE INFORMAÇÃO LILACS E A LINGUAGEM DeCS NA PERSPECTIVA DA COMUNICAÇÃO CIENTÍFICA}

Visando a qualidade e o aprimoramento dos serviços de atendimento aos usuários da comunidade científica da área de saúde, a BIREME desenvolve um trabalho cooperativo com mais de 500 bibliotecas - centros cooperantes - integrantes do Sistema Latino-Americano e do 
Caribe de Informação em Saúde, representado por 37 países, na indexação de documentos em fontes de informação como a base de dados LILACS.

O sistema de informação/base de dados LILACS tem por finalidade o controle da produção bibliográfica e a disseminação da literatura científica latino-americana e caribenha na área de Ciências da Saúde (Medicina, Medicina Veterinária, Odontologia, Saúde Pública, Psicologia, Fonoaudiologia, entre outras), com a descrição formal e de conteúdo de teses, livros, capítulos de livros, anais de congressos ou conferências, relatórios técnico-científicos, artigos de revistas, materiais não convencionais etc., produzidos por autores latino-americanos e caribenhos, publicada nos países da região, a partir de 1982.

Essa base de dados originou-se da base de dados do Index Medicus LatinoAmericano (IMLA), iniciada em 1979. Posteriormente, a BIREME desenvolveu a Metodologia LILACS, baseada na experiência adquirida pela base IMLA e compatível com as já existentes e utilizadas pelos centros cooperantes da rede para a entrada e recuperação de informações.

A linguagem de recuperação adotada pelo sistema LILACS é o Vocabulário Estruturado DeCS - Descritores em Ciências da Saúde, sendo sua abrangência temática específica da área de ciências da saúde. “Os vocabulários estruturados são coleções de termos, organizados segundo uma metodologia na qual é possível especificar as relações entre conceitos com o propósito de facilitar o acesso à informação”. (BIREME, 2004).

O Vocabulário Estruturado DeCS tem a finalidade de ser a linguagem única para a indexação e recuperação de documentos na base de dados LILACS pelo Sistema LatinoAmericano e do Caribe de Informação em Ciências da Saúde.

Para a sua elaboração, tomou-se como base a linguagem documentária $\mathrm{MeSH}$ Medical Subject Headings, produzida pela United States National Library of Medicine NLM, publicado em 1960 e atualizado em 1963, para a indexação e recuperação de artigos de periódicos publicados nos Estados Unidos e em mais de 70 países, disponibilizados na base de dados MEDLINE (UNITED STATES NATIONAL LIBRARY OF MEDICINE, 2005).

A $1^{\mathrm{a}}$ edição do DeCS é datada de 1987, no formato impresso, apresentada em dois volumes constituídos pelas listas alfabética e hierárquica, nos idiomas português e espanhol, ocorrendo sucessivamente, a publicação de duas outras edições.

A partir do ano de 1999, o Vocabulário DeCS, formado pelas listas alfabética, permutada e hierárquica, foi disponibilizado na versão online (DeCS Home), via internet, no endereço eletrônico http://decs.bvs.br/. 
Dentro das linguagens documentárias alfabéticas, o DeCS, tal qual o MeSH, é considerado um tesauro que, embora seguindo a tradição dos sistemas de classificação e das listas de cabeçalhos de assunto, foi transformando-se em um vocabulário controlado especializado, sem, contudo, abandonar as estruturas dos referidos sistemas de classificação das quais é originário.

Sua estrutura hierárquica é fundamentada na divisão do conhecimento em classes e subclasses decimais, respeitando as ligações conceituais e semânticas, e seus termos são apresentados em uma estrutura híbrida de pré e pós-coordenação para a indexação e recuperação de informações.

Assim, o DeCS é um vocabulário traduzido do MeSH para os idiomas português e espanhol, apresentando os termos que o compõem nos três idiomas (inglês, português e espanhol). A estrutura e a organização dos descritores foram mantidas tal como disponibilizadas no $\mathrm{MeSH}$, estando os grandes assuntos apresentados em categorias.

Para atender às necessidades de indexação e recuperação da informação de centros cooperantes de algumas áreas não representativas e/ou inexploradas pela literatura e, conseqüentemente, pelas bases de dados internacionais, foram necessárias a criação de duas categorias representativas das áreas temáticas de Saúde Pública e Homeopatia, não existentes na linguagem $\mathrm{MeSH}$.

O DeCS é uma linguagem poli-hierárquica que possibilita um mesmo descritor estar hierarquizado em mais de uma categoria. As relações lógico-semânticas estão presentes entre os termos, porém nem sempre de acordo com as diretrizes internacionais estabelecidas (AUSTIN; DALE, 1993).

Com relação à área deste estudo, a Fonoaudiologia, esta é formada pelas especialidades de Linguagem, Audiologia, Voz e Motricidade Oral. (CONSELHO FEDERAL DE FONOAUDIOLOGIA, 2001). O Vocabulário DeCS não possui uma categoria específica para a Fonoaudiologia, sendo assim, ela encontra-se hierarquizada dentro da categoria de Saúde Pública. Todavia, pelo fato de ser uma área multidisciplinar, seus termos também estão hierarquizados em várias outras categorias do vocabulário, sendo as principais a Anatomia, Doenças, Técnicas e Equipamentos, Psicologia e Psiquiatria, Antropologia, Educação, Sociologia e Fenômenos Sociais, entre outras.

O termo Fonoaudiologia é inexistente nos Estados Unidos e na Europa pelo fato da estrutura da área ser diferente em relação aos países da América Latina, onde as semelhanças são mais evidentes. O termo Speech-Language Pathology engloba os estudos das especialidades da Fala (Speech), Linguagem (Language), Voz (Voice) e Motricidade Oral 
(termo também inexistente nos Estados Unidos - Oral Motricy). A Audiologia é uma especialidade independente e representada pelo termo Audiology.

Nesse sentido, o termo Fonoaudiologia, não existente no vocabulário da língua inglesa, foi traduzido no DeCS como Speech, Language and Hearing Sciences e de acordo com a literatura fonoaudiológica brasileira, ainda há muita controvérsia se essa seria a tradução mais adequada.

Dentro desse contexto, Hudon (1997) apresenta considerações sobre a elaboração de tesauros multilingües tendo em vista as várias culturas que estes podem apresentar.

Conforme a autora, a decisão mais importante que se deve tomar no momento da elaboração de tesauros multilingües é sobre a forma de tratamento dado às estruturas semânticas. Assim, apresenta dois tipos de estruturas a serem consideradas: a estrutura semântica idêntica e simétrica e a estrutura semântica não-idêntica e assimétrica.

Considerando-se as diferenças culturais que possam ocorrer e que um termo possa existir em uma língua e não haver um correspondente em outra, com a adoção de estruturas semânticas idênticas e simétricas, cada termo deverá possuir um correspondente nas diversas línguas do tesauro e isso pode ocasionar hierarquias semanticamente incorretas ou ilógicas.

Nas estruturas semânticas não-idênticas e assimétricas, a variação do número de descritores em cada versão lingüística de um tesauro é permitida, visto que os conceitos terminológicos que existem em uma cultura nem sempre são representáveis em outra cultura. Dessa forma, um tesauro multilingüe em que às estruturas são permitidas diferir, é mais provável que o universo cultural dos conceitos, e conseqüentemente dos termos, represente as diversas culturas lingüísticas, constituindo-se assim, um tesauro multilingüe e multicultural.

Portanto, o usuário/pesquisador utilizando uma linguagem documentária representativa de sua cultura terminológica na realização de suas buscas bibliográficas, terá mais condições de obter resultados úteis e pertinentes à sua atividade investigativa que possibilitarão assisti-lo nas tomadas de decisões, nas resoluções de problemas e na geração de novos conhecimentos.

\section{AVALIAÇÃO DE LINGUAGEM DOCUMENTÁRIA: pressupostos teóricos}

O desempenho de uma linguagem documentária na recuperação da informação é um fator essencial na obtenção de informações satisfatórias para o usuário/pesquisador, 
possibilitando a realização de investigações que contribuam para o desenvolvimento da Fonoaudiologia e da ciência brasileira, visando a melhoria de vida da coletividade.

A busca da informação desejada pelo usuário e sua obtenção, de acordo com o assunto da pesquisa, é a realização de um trabalho integrado entre o sistema, a linguagem documentária e o próprio usuário.

Nesse contexto, muitos estudos têm-se realizado a respeito de avaliação de linguagens documentárias pelo uso, pela forma e/ou pelo conteúdo com aplicação de abordagens quantitativas, qualitativas e/ou cognitivas, com o intuito de verificar-se o seu desempenho em Sistemas de Informação na recuperação de informação.

Destacam-se, nesse campo de estudo, pesquisadores como Cleverdon (1964) e Lancaster (2004) no desenvolvimento de trabalhos com a definição de critérios e dos índices quantitativos de previsão e revocação que contribuíram com o estabelecimento de parâmetros metodológicos para a avaliação de linguagens.

Dentro dessa abordagem quantitativa, também merecem especial atenção outros trabalhos internacionais sobre a avaliação de linguagens pelo uso como os testes da ASTIA (Armed Services Technical Information Agency), os Projetos Cranfield I e II, o programa desenvolvido por F. W. Lancaster para a avaliação de desempenho do Sistema Medlars, o teste de Aberystwyth envolvendo estudos sobre linguagens natural e controladas e os estudos de Indexação Sistemática de J. Kaiser, complementado, posteriormente, pelos testes realizados pela WRU (Western Reserve University), tendo como ponto focal a avaliação formal de linguagens. (FOSKETT, 1973; MELO, 1994).

As pesquisas no Brasil também apresentaram trabalhos muito importantes sobre a avaliação formal de linguagens como o realizado por Lara (1993) em sua dissertação de mestrado e o de Strehl (1998) com a análise de um vocabulário controlado utilizado numa base de dados de uma biblioteca universitária de artes.

Na área de Saúde, o Vocabulário Estruturado DeCS também foi objeto de estudos de alguns pesquisadores internacionais e nacionais, na verificação de sua "performance” e de sua estrutura na indexação e recuperação de informação em Sistemas de Informação. Assim, apresentam-se os trabalhos desenvolvidos pelos estudiosos cubanos Valdés de Abreu (1996), Rodriguéz Camiño (1998), Jiménez Miranda (1998, 2002) e Pellizzon (2004), bem como o dos pesquisadores argentinos Otero et al. (2004).

O estudo brasileiro de Santos (2002) também contribuiu para o aprimoramento da linguagem DeCS com o desenvolvimento de um estudo de avaliação na categoria de Saúde Pública, utilizada para a indexação e recuperação da informação na Base de Dados AdSaúde 
(Administração em Saúde), do Sistema Especializado em Administração em Saúde do Sistema BIREME.

No âmbito das pesquisas de avaliação centrada no usuário, os estudos qualitativos baseados em percepções e/ou opiniões devem ser considerados fundamentais, visto que as atitudes e as opiniões emitidas pelo usuário devem ser consideradas de suma importância para o aprimoramento de serviços de recuperação de informação e, conseqüentemente, das linguagens documentárias utilizadas pelo sistema.

O desenvolvimento dessas pesquisas, utilizando abordagens cognitivas, foram realizadas em diversas áreas do conhecimento, principalmente na área da Ciência da Informação, a partir da década de 1970.

Os trabalhos pioneiros de Ericsson e $\operatorname{Simon}^{2}$ (1987 apud FUJTTA, NARDI e FAGUNDES, 2003, p. 144) com a utilização da técnica do protocolo verbal na observação da atividade de leitura também colaboraram com resultados promissores para os estudos de observação.

Para a verificação da eficácia da atividade de recuperação da informação e a interação do usuário, em especial os aspectos cognitivos do processo, Ingwersen (1982) realizou uma avaliação qualitativa utilizando o protocolo verbal no serviço de referência de uma biblioteca pública.

Segundo Ingwersen (1982), os sujeitos eram formados por treze bibliotecários, sendo sete bibliotecários ocupantes de cargos de direção, três bibliotecários de referência e três que trabalhavam em ambas as atividades. Os trezes possuíam vários anos de experiência na biblioteca, e as bibliotecas escolhidas cobriam tanto cidades grandes quanto as de áreas rurais. Os cinco usuários que participaram foram escolhidos entre as pessoas com uma experiência limitada de biblioteca: quatro eram técnicos e um era estudante de faculdade.

Muita atenção foi dada à instrução dos sujeitos antes dos testes, isto é, a realização da familiarização dos 13 sujeitos com a técnica, treinamentos sobre como "pensar alto”, isto é, como externar seus pensamentos no momento da execução da atividade de recuperação da informação, durante a gravação e, também, sobre a utilização do Sistema de Informação. Complementando a aplicação da técnica, o autor observou o comportamento dos sujeitos durante todo o processo. Realizou estudo sobre a negociação que ocorreu durante a pesquisa nos Sistemas de Informação, entre o usuário e o bibliotecário.

\footnotetext{
${ }^{2}$ ERICSSON, k. A.; SIMON, H. A. Verbal reports on thinking. In: FAERCH, C.; KASPER, G. (Ed). Introspection in second language research. Clevedon: Multilingual Matters, 1987. p. 24-53 apud FUJITA; NARDI; FAGUNDES, 2003, 144.
} 
Sete gravações foram feitas e cobriram a busca do usuário, seguida pela negociação e pela busca da informação. Cada gravação foi baseada na necessidade do usuário participante sobre os assuntos: 1) as identidades da álgebra booleana na forma apropriada para a aplicação, criação e reparo de circuitos nos computadores; 2) literatura não tendenciosa sobre o pacto de 1939, alemão-soviético na forma de fontes de informação, correspondências e textos sobre o pacto, com especial consideração em esclarecer de que lado originou-se a iniciativa.

Cada registro foi transcrito em um protocolo verbal, tendo sido incluídos alguns fatos ocorridos bem como as observações referentes ao comportamento dos sujeitos envolvidos. Comentários adicionais de sua própria observação foram incorporados.

A técnica introspectiva do protocolo verbal ou "pensar alto” (Thinking aloud) é aplicada a estudos de avaliação qualitativa onde os sujeitos, em voz alta, expressam o que pensam e o que ocorre em suas mentes durante a execução de uma tarefa. Essas declarações são gravadas, observando-se também o comportamento dos sujeitos como expressões faciais (gestos e movimentos dos olhos). Dessa maneira, a linguagem do pensamento realiza muitos processos cognitivos como a percepção e o raciocínio.

Segundo Cohen $^{3}$ (1984 apud NARDI, 1999, p. 123), as técnicas introspectivas são “medidas mentalísticas” apresentando-se sob três formas básicas: dados provenientes de autorelato, auto-observação e auto-revelação.

[... ] o auto-relato refere-se a declarações dos indivíduos sobre como acreditam que realizam certas tarefas, fornecidas em situações independentes da situação de realização efetiva da tarefa em questão. A auto-observação refere-se a inspeções de comportamentos específicos durante a realização de uma tarefa ou enquanto a informação ainda está sob o foco da atenção [...] ou após o evento, retrospectivamente. A auto revelação não é nem descrição nem inspeção de comportamentos específicos; é um "pensar alto" durante a realização da tarefa; o pensamento é direta e automaticamente externalizado; os dados são obtidos sem análise nem edição.

No Brasil, entre vários estudos desenvolvidos com o emprego dessa técnica, a dissertação de mestrado de $\operatorname{Nardi}^{4}$ (1993 apud FUJITA, NARDI e FAGUNDES, 2003, p. 146) “abriu a possibilidade de uso de um instrumento de coleta de dados, também, para a observação da leitura documentária dentro do Grupo de Pesquisa a Análise Documentária da UNESP - Campus de Marília”. A pesquisa pioneira no Brasil, desenvolvida por Fujita, Nardi

\footnotetext{
${ }^{3}$ COHEN, A. D. Using verbal reports on research on language learning. In FAERCH; KASPER (ED). Introspection in second language research. Clevedon: Multilingual Matters, 1987, apud NARDI, 1999, p. 123.

${ }^{4}$ NARDI, M. I. A . As expressões metafóricas na compreensão de texto escrito em língua estrangeira. 1993. Dissertação (Mestrado em Lingüística Aplicada ao Ensino de Línguas) - Pontifícia Universidade Católica, São Paulo apud FUJITA; NARDI; FAGUNDES, 2003, p. 146.
} 
e Fagundes (2003), na observação da leitura documentária, apresenta excelentes resultados no uso do protocolo verbal, demonstrando novos aspectos da leitura documentária entre outras contribuições.

Com a utilização da técnica do protocolo verbal, os usuários “pensam em voz alta”, emitindo suas opiniões e comentários acerca do objeto avaliado, realizando uma avaliação cooperativa e participativa: usuários participam da identificação e entendimento de problemas de recuperação da informação e utilização do sistema no seu próprio ambiente de trabalho.

Por considerar de fundamental importância a observação do usuário para a realização da avaliação da linguagem documentária DeCS, possibilitando uma análise mais efetiva e fiel de suas necessidades informacionais, e pela verificação de importantes resultados obtidos em pesquisas nacionais e internacionais realizadas na área da Ciência da Informação, utilizou-se neste estudo a técnica do protocolo verbal ou "pensar alto" (thinking aloud) como instrumento de coleta de dados.

Essa avaliação foi desenvolvida de acordo com os critérios metodológicos apresentados por Ingwersen (1982) e Fujita, Nardi e Fagundes (2003), realizando-se alguns ajustes à realidade investigativa, quando necessário. A forma da técnica aplicada foi o protocolo verbal individual com “escora”, isto é, com negociação - interferência da observadora/pesquisadora junto ao usuário/pesquisador (sujeito) durante a aplicação da técnica.

Segundo Nardi (1999), o termo “escora” deve-se ao fato da existência dessa interação entre o pesquisador e o sujeito, em alguns momentos da realização do protocolo verbal, denominado assim pela própria autora como "protocolos interativos" ou "protocolos com escora”.

Conforme Spradley (1980), a observação participante pode ser entendida dentro de três universos conceituais: a participação passiva onde o pesquisador se comporta como mero observador da situação avaliativa; a participação moderada tendo o pesquisador à função alternada de observador e participante ativo e a participação ativa onde o pesquisador desempenha uma função de participante no mais alto grau de atuação, isto é, procura realizar a atividade que os outros participantes estão fazendo (realiza a atividade avaliativa e analisa os dados do grupo).

Relativamente a esse contexto, a participação, neste estudo, foi a do tipo participante moderada, intervindo nos momentos de recuperação de informações, manifestando-nos aos sujeitos sobre o grau de satisfação dos resultados obtidos, se os termos utilizados na pesquisa 
corresponderam efetivamente a sua necessidade informacional e se os termos não poderiam ter sido substituídos por outros disponíveis na linguagem documentária utilizada.

Todavia, foi necessário um planejamento prévio das etapas de trabalho que seriam desenvolvidas, bem como a adoção de alguns procedimentos que antecederiam a aplicação efetiva da técnica do protocolo verbal para a coleta de dados.

\section{METODOLOGIA}

Os procedimentos de aplicação do protocolo verbal iniciaram-se com a definição dos sujeitos do estudo que recaiu sobre os docentes/pesquisadores do Departamento de Fonoaudiologia da Faculdade de Odontologia de Bauru da Universidade de São Paulo FOBUSP, visando atender suas necessidades na realização de pesquisas clínicas, básicas e aplicadas para o desenvolvimento e aprimoramento de técnicas/métodos diagnósticos e terapêuticos para o melhor atendimento da sociedade em geral.

A aplicação dessa técnica foi realizada no próprio ambiente de trabalho dos sujeitos, tendo sido verificado, anteriormente, o conhecimento de cada um deles no acesso ao sistema LILACS, bem como na utilização da linguagem DeCS.

A partir de então, foram selecionados quatro sujeitos, representantes das quatro especialidades formadoras da área de Fonoaudiologia: Linguagem, Audiologia, Voz e Motricidade Oral, e observados, com protocolo verbal, na tarefa de realizar a recuperação de informações, no campo de descritor de assunto da interface de busca da base de dados LILACS utilizando a linguagem DeCS. Os temas pesquisados foram definidos pelos próprios sujeitos.

Dessa forma, a coleta dos dados foi proveniente das declarações efetuadas pelos sujeitos durante a realização da referida atividade avaliada, com a interação e intervenção moderada da observadora/pesquisadora.

As opiniões emitidas pelos sujeitos foram gravadas e, posteriormente, transcritas para a análise dos dados coletados. 


\section{RESULTADOS E DISCUSSÃO}

Os dados coletados pelas declarações dos sujeitos participantes deste estudo proporcionaram subsídios para a realização da análise dos resultados sobre a realidade temática atual dos termos correspondentes ao campo conceitual de Fonoaudiologia do Vocabulário DeCS.

A análise das transcrições dos protocolos verbais demonstrou que a metodologia empregada produziu resultados satisfatórios por possibilitar a coleta, em tempo real, das declarações dos sujeitos (usuários/pesquisadores) sobre o desempenho da linguagem DeCS utilizada para a recuperação de informações no sistema LILACS.

O estudo realizou uma reflexão sobre as declarações emitidas pelos quatro sujeitos participantes, com o intuito de proporcionar as condições necessárias para eleger-se indicadores de qualidade que irão delinear as estratégias para o aprimoramento do Vocabulário DeCS na área de Fonoaudiologia.

Os sujeitos participantes apontaram as seguintes ocorrências como responsáveis pela ineficácia da linguagem DeCS, durante as buscas realizadas no sistema de informação LILACS: a insuficiência de termos genéricos e/ou específicos representativos da área de Fonoaudiologia; a inconsistência nas relações lógico-semânticas, existente entre os termos; a hierarquização de termos em categorias de assuntos não equivalentes aos seus conceitos; a necessidade de atualização constante dos termos disponíveis na linguagem com relação à terminologia encontrada na literatura científica da área e adotada pelos especialistas; a tradução de diversos termos, notas de escopo e definições, da língua inglesa para a portuguesa, e desta para a inglesa, que não correspondem ao equivalente utilizado na área de Fonoaudiologia; a correspondência indevida de termos aos significados propostos e apresentados pela linguagem e equívocos ocorridos na indexação. Além disso, os sujeitos, também, manifestaram as suas insatisfações com os resultados obtidos, por meio de suas atitudes e sentimentos como irritabilidade, frustração e indignação, expressos durante a recuperação da informação com a utilização do Vocabulário DeCS.

\section{CONSIDERAÇÕES FINAIS}

A opção por utilizar um instrumento de coleta de dados introspectivo, centrado no usuário, permitiu a realização de uma análise dos resultados muito mais verídica e confiável 
em relação aos problemas apresentados pela linguagem documentária, pelo fato de o usuário ser o "elemento" fundamental da triangulação: sistema de informação (intermediado pela própria linguagem como o instrumento de comunicação) - informação documentária usuário.

Segundo Lancaster (1987), a garantia literária fundamenta-se no sentido de que termo só se justifica quando se tem conhecimento da existência de literatura sobre o assunto; a garantia de uso é referente aos termos coletados a partir das solicitações de buscas realizadas pelos usuários.

Além disso, a elaboração dessa linguagem documentária deve estar de acordo com as diretrizes e normas pré-estabelecidas e disponíveis na área, quanto à forma de apresentação de seus termos e de sua estrutura lógico-semântica, entre outros segmentos necessários para sua consistência no momento da recuperação da informação.

Confirmou-se, também, neste estudo a idéia de que a linguagem documentária, enquanto veículo de comunicação, deve representar os campos conceituais respeitando a cultura da comunidade à qual a linguagem serve. Um sistema de organização e/ou representação do conhecimento deve adotar princípios que garantam a eqüidade. Desse modo, uma cultura não deve sobrepor-se a outra. A dominação pode gerar exclusões que comprometem, no caso dos sistemas de informação, a circulação do conhecimento. (BEGHTOL, 2002).

De acordo com os conceitos de Hudon (1997), a linguagem DeCS apresenta-se seguindo os princípios dos tesauros multilingües de estruturas semânticas idênticas e simétricas.

Dentro desse contexto, a pesquisa mostrou que a linguagem DeCS deve ser elaborada de acordo com as estruturas semânticas não-idênticas e assimétricas.

Para tanto, é necessário fazer a revisão da área de Fonoaudiologia no DeCS, para torná-la compatível com a realidade cultural e terminológica dos pesquisadores brasileiros.

Além disso, as ocorrências apontadas pelos sujeitos participantes e apresentadas nesse estudo não corresponderam aos conceitos apresentados pela literatura, fornecendo assim, os subsídios necessários para a identificação dos principais elementos responsáveis pela ineficácia da linguagem.

Nesse sentido e, de acordo com a situação atual em que se encontram os termos de Fonoaudiologia constituintes da linguagem documentária DeCS, recomenda-se a BIREME a construção de uma categoria específica para a área de Fonoaudiologia, visto que esse campo temático tem características específicas dentro da área das ciências da saúde brasileira, 
possibilitando a correspondência entre a representação terminológica e as necessidades dos usuários/pesquisadores brasileiros para a recuperação de informações que contribuem para a criação do conhecimento e para a construção da ciência brasileira.

Considera-se, também, essa medida totalmente viável pelo fato da linguagem DeCS já apresentar adaptações necessárias com a inclusão das categorias de Saúde Pública e Homeopatia inexistentes na linguagem documentária de origem, o MeSH.

Dentro do contexto do trabalho cooperativo empregado pela BIREME no desenvolvimento de seus serviços por meio da comunidade especializada no gerenciamento, tratamento e disseminação da informação - os centros cooperantes -, a elaboração de uma categoria específica para a área de Fonoaudiologia deve ser desenvolvida dentro de uma filosofia de trabalho participativo e integrado.

Assim, os especialistas/pesquisadores da área, os profissionais da informação que possuam experiência na atividade de indexação e/ou recuperação da informação nessa temática, bem como conhecimentos sobre a elaboração linguagens documentárias e as Sociedades de Pesquisa, como a Sociedade Brasileira de Fonoaudiologia (SBFa) são recursos humanos e institucionais que reúnem todas as condições para o desenvolvimento dessa categoria disciplinar.

Para tanto, alguns indicadores de qualidade foram delineados como elementos norteadores para a elaboração da categoria de Fonoaudiologia, dentro da estrutura/relação poli-hierárquica que o Vocabulário possui:

a) Levantamento e padronização da terminologia da área de Fonoaudiologia utilizada pelos especialistas (usuários/pesquisadores), Sociedades de Pesquisas e pela literatura científica que represente da maneira mais fiel e amplamente possível as especialidades de Linguagem, Audiologia, Voz e Motricidade Oral, dentro dos princípios de generalidade e especificidade dos termos e das diretrizes e normas existentes para tal;

b) Atualização dos descritores já existentes, para a sua incorporação, quanto à sua terminologia e definições, indo ao encontro da realidade científica fonoaudiológica brasileira;

c) Revisão das relações lógico-semânticas dos termos já existentes e estabelecimento dessas relações com todos os novos termos que vierem a integrar-se ao vocabulário;

d) Controle mais efetivo dos termos sinônimos e quase-sinônimos, evitando-se a dispersão temática, proporcionando exatidão na indexação de conteúdo e uma recuperação mais eficaz da informação; 
e) Expansão das áreas de Educação, Psicologia, Lingüística, Neurolinguística, Medicina (Otorrinolaringologia), Odontologia, Física e Saúde Pública para atender às necessidades de relacionamento temático desses campos conceituais com a Fonoaudiologia, visando a característica multidisciplinar que a área possui;

f) Levantamento das necessidades de incorporação de outros termos das áreas afins da Fonoaudiologia como: anatomia, fisiologia, bioquímica, entre outras;

g) Revisão da tradução de todos os termos existentes no vocabulário DeCS - dos idiomas inglês e espanhol para o português -, visando a devida adequação conceitual dos termos empregados pela literatura fonoaudiológica brasileira;

h) Realização da versão do português para o inglês e o espanhol em todos os novos termos, de acordo com o uso terminológico empregado pela comunidade científica norteamericana e latino-americana;

i) Revisão das "Notas de Definição", com a verificação dos conceitos que cada termo possui e o estabelecimento dessas notas/definição para todos os termos que integrarão a categoria;

j) Revisão das “Notas de Indexação”, e o estabelecimento dessas, quando necessário, aos novos termos;

k) Estabelecimento dos qualificadores que serão considerados “permitidos para uso” com os termos novos.

Com a adoção desses indicadores de qualidade, ter-se-á a elaboração de uma linguagem documentária estruturada e representativa da cultura de seu sistema de informação que proporcionará uma consistência maior das informações armazenadas e recuperadas, bem como o aumento da eficácia do índice temático e, conseqüentemente, da recuperação da informação (SANTOS, 2002).

Essa medida possibilitará, também, um melhor desempenho da terminologia latinoamericana junto ao projeto de desenvolvimento de uma terminologia unificada e rede semântica em saúde, a Unified Medical Language System (UMLS), http://www.nlm.nih.gov/research/umls/, proposto pela United States National Library of Medicine, tendo a BIREME a responsabilidade da atualização e envio dos termos em português e espanhol.

\section{REFERÊNCIAS}


ANDRADE, C. R. F. de. Fonoaudiologia preventiva: teoria e vocabulário técnico-científico. São Paulo: Lovise, 1996. 165 p.

AUSTIN, D.; DALE, P. Diretrizes para o estabelecimento e desenvolvimento de tesauros monolingues. Tradução de Bianca Amaro de Melo. Brasília: IBICT, 1993. 78 p.

BEGHTOL, C. Universal concepts, cultural warrant, and cultural hospitality. In: LÓPEZHUERTAS, M. J.; MUNOZ FÉRNANDEZ, M. J.; FRANCISCO, J. (Ed.). Challenges in knowledge representation and organization for the 21st century: integration of knowledge across boundaries: proceedings of the International ISKO Conference, 7th., 2002, Granada. Würzburg: Indeks Verlag, 2002.

BIREME - CENTRO LATINO-AMERICANO E DO CARIBE DE INFORMAÇÃO EM CIÊNCIAS DA SAÚDE. Fundamentos, missão, objetivos, estrutura organizacional. Disponível em: <http://www.bireme.br/bvs/bireme/P/objetivos.htm>. Acesso em: 29 dez. 2004a.

BIREME - CENTRO LATINO-AMERICANO E DO CARIBE DE INFORMAÇÃO EM CIÊNCIAS DA SAÚDE. O que são vocabulários estruturados? Disponível em: http://decs.bvs.br/P/aboutvocabp.htm. Acesso em: 30 set. 2004b.

CONFERÊNCIA NACIONAL DE CIÊNCIA, TECNOLOGIA E INOVAÇÃO EM SAÚDE, 2., 2004, Brasília. Manual. Brasília: Conselho Nacional de Saúde, Ministério da Saúde, 2004. $164 \mathrm{p}$.

CONSELHO FEDERAL DE FONOAUDIOLOGIA. Resolução CFFa n⿳0 269, de 03 de

março de 2001: dispõe sobre as especialidades reconhecidas pelo Conselho Federal de

Fonoaudiologia, e dá outras providências. Disponível em:

http://www.Fonoaudiologia.org.br/resolu/RESOL269.HTM. Acesso em: 02 fev. 2005.

CLEVERDON, C. W. Evaluation of operational information retrieval systems. Part 1: identification of criteria. Cranfield: College of Aeronautics, 1964.

FOSKETT, A.C. Pesquisa em recuperação da informação. In: __. A abordagem temática da informação. Tradução de Antonio Agenor Briquet de Lemos. São Paulo: Polígono, 1973. p. 371-391.

FUJITA, M. S L.; NARDI, M. I. A.; FAGUNDES, S. A . A observação da leitura documentária por meio de protocolo verbal. In: RODRIGUES, G. M.; LOPES, I. L. (Org).

Organização e representação do conhecimento na perspectiva da ciência da informação. Brasília: Thesaurus, 2003. p. 141-178. (Estudos avançados em ciência da informação, v. 2).

HUDON, M. Multilingual thesaurus construction: integranting the views of different cultures in one gateway to knowledge and concepts. Knowledge Organization, Frankfurt, v. 24, n. 2, p. 84-91, 1997.

INGWERSEN, P. Search procedures in the library: analysed from the cognitive point of review. Journal of Documentation, London, v. 38, n. 3, p. 165-191, Sept. 1982.

JIMÉNEZ MIRANDA, J. Acceso a MEDLINE y LILACS mediante el MeSH e el DeCS.

ACIMED, Cidadd de Habana, v. 6, n. 3, p. 153-162, sep./dic. 1998. Disponível em:

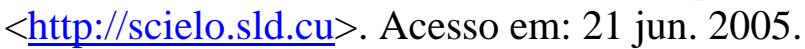

JIMÉNEZ MIRANDA, J. La indización em el Sistema Nacional de Información de Ciencias Médicas: Parte II Descriptores en Ciencias de la Salud (DeCS). ACIMED, La Habana, v. 10, n. 4, jul./ago. 2002. Disponível em: <http://scielo.sld.cu>.. Acesso em: 21 jun. 2005.

LANCASTER, F. W. Construção e uso de tesauros: curso condensado. Tradução de César Almeida de Meneses Silva. Brasília: IBICT, 1987. 114 p.

LANCASTER, F. W. Indexação e resumos: teoria e prática. Tradução de Antonio Agenor Briquet de Lemos. 2. ed. Brasília: Briquet de Lemos, 2004. 452 p. 
LARA, M. L. G. de. A representação documentária: em jogo a significação. 1993. 133 f. Dissertação (Mestrado) - Departamento de Biblioteconomia e Documentação, Escola de Comunicações e Artes, Universidade de São Paulo, São Paulo.

MARCHESAN, I. Q.; ZORZI, J. L.; GOMES, I. C. D. (Org.). Breve relato da história da fonoaudiologia no Brasil. In: Tópicos em fonoaudiologia: 1997/1998. São Pualo: Lovise, 1998. v. 4.

MELO, L. B. Avaliação de sistemas de recuperação de informação: breve retrospectiva dos principais projetos. Cadernos BAD, Coimbra, v. 2, p. 29-46, 1994.

NARDI, M. I. A. A metáfora e a prática de leitura como evento social: instrumentos do pensar a biblioteconomia do futuro. 1999. 268 f. Tese (Doutorado em Lingüística Aplicada e Estudos da Linguagem) - Pontifícia Universidade Católica de São Paulo, São Paulo.

OTERO, P. et al. Evolution of medical informatics in bibliographic databases. Medinfo, Amsterdam, v. 11, Pt. 1, p. 301-305, 2004.

PELLIZZON, R. de F. Pesquisa na área de saúde: 1 - base de dados DeCS (Descritores em Ciências da Saúde). Acta Cirúrgica Brasileira, São Paulo, v. 19, n. 1, p. 153-163, mar./abr. 2004. Disponível em: <http://www.scielo.br>. Acesso em: 29 set. 2004.

RODRÍGUEZ CAMIÑO, R. MeSH o DeCS: algunas consideraciones sobre la indización biomédica. ACIMED, La Habana, v. 6, n. 3, p. 163-170, sep./dic. 1998. Disponível em:

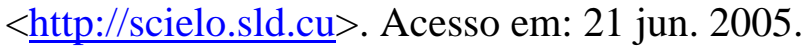

SANTOS, C. A. C. M. dos. Linguagens documentárias e codificação da informação: estudo de vocabulário da área de saúde. 2002. 70 f. Dissertação (Mestrado) - Escola de Comunicação e Artes, Universidade de São Paulo, São Paulo.

SPRADLEY, J. P. Participant observation. New York: Holt, Rinehart \& Winston, c1980. $195 \mathrm{p}$.

STREHL, L. Avaliação da consistência da indexação realizada em uma biblioteca

universitária de artes. Ciência da Informação, Brasília, v. 27, n. 3, p. 329-355, set./ dez. 1998.

UNITED STATES NATIONAL LIBRARY OF MEDICINE. Medical subject headingd MeSH. Disponível em: http://www.nlm.nih.gov/mesh/MBrowser.html. Acesso em: 13 mar. 2005.

VALDÉS ABREU, M. de la C. Necesidad de las notas de alcance de los calificadores en la "Introducción" del tesauro Descriptores en Ciencias de la salud (DeCS). ACIMED, Ciudad de Habana, v. 4, n. 1, p. 23-28, ene./abr. 1996. Disponível em: <http://scielo.sld.cu $>$. Acesso em: 21 jun. 2005.

\footnotetext{
ABSTRACT

The proposition of this study is to assess, by observing the user, the DeCS - Health Sciences Descriptors indexing language, in the Speech-Language Pathology and Audiology area, utilized for the retrieval of information in the LILACS System - Latin American and Caribbean Health Sciences -, produced by BIREME - Latin-American and Caribbean Health Sciences Information Center, utilizing the verbal protocol technique, for the fact of the researchers of the area of Speech-Language Pathology and Audiology to need an information system that uses a indexing language that represents the values and the culture of this system where it is inserted. With this proposal, it is had for objective to contribute for the improvement of the indexing language DeCS, being aimed at better terminological representation in the area of the Brazilian Speech-Language Patholoy and Audiology as a determinative factor for the development of scientific research of quality. The used methodological approach was the qualitative-cognitive one, has been the technique verbal
} 
protocol applied in the researchers (subjects) of the Speech-Language Pathology and Audiology Department at the Dental School - University of São Paulo - FOB-USP, at Bauru, SP, representing the four forming specialties in the area, i.e., Language, Audiology, Voice and Oral Motricity. The analysis of the transcriptions these protocols demonstrated that the methodology employed had good performance, as well as made possible a reflection on the declarations issued showing that the DeCS language, in Speech- Language Pathology and Audiology, led the searches to unsatisfactory results as to information retrieval from the following relevant aspects as insufficiency of generic and/or specific terms representative of the Speech-Language Pathology and Audiology area; terms available in the language regarding the terminology found in the scientific literature of the area and adopted by specialists need to be updated; ranking of terms in subject categories not equivalent to their concepts; translation of various terms from the English language into the Portuguese language and from this into English which do not correspond to the equivalent used in the area of Speech-Language Pathology and Audiology, among others. It is concluded that becomes necessary the improvement of the DeCS indexing language in the field of Speech-Language Pathology and Audiology and therefore, it is recommended that the BIREME elaborate a specific category these area for the its effective terminological representation, with the utilized in the literature and the Brazilian scientific community.

KEYWORDS: Indexing Language. Evaluation. Information System. Information Retrieval. Verbal Protocol. Speech-Language Pathology and Audiology.

Originais recebidos em 19/07/2005 